\title{
Los inicios de la mujer en la práctica del ajedrez en España (1922-1935)
}

\author{
Jordi BRASÓ RIUS \\ Universidad de Barcelona \\ jbrasorius@ub.edu
}

Recibido: Enero 2016

Aceptado: Noviembre 2016

\section{RESUMEN}

A finales del s. XIX se sitúan los primeros movimientos feministas. Uno de los campos de acción se relacionó con la práctica del ajedrez, objeto de estudio del presente artículo. Mediante el uso de una metodología basada en el análisis hermenéutico de las principales fuentes primarias de la época, se concluye que la introducción de la mujer en el juego surge en Inglaterra a mediados del s. XIX. En España, la práctica ajedrecística la encabezó Barcelona, durante los inicios del s. XX, aunque no aparece un movimiento significativo hasta la década de los años 30.

Palabras clave: ajedrez, Barcelona, Escuela del Mar, España, mujer.

The beginnings of women in the practice of chess in Spain (1922-1935)

\begin{abstract}
Early feminist movements in different fields won't appear until the end of nineteenth century. One of these fields is sport, which includes chess: the object of study of this article. Using a methodology based on hermeneutic analysis of the principal information sources, it is possible to conclude that the introduction of women in chess associations toke place in the middle of the nineteenth century in England. In Spain, Barcelona led this practice. Spanish chess societies include women at the beginnings of Twentieth century, but it was not until the thirties when significant female movements appear in this sport.
\end{abstract}

Keywords: Barcelona, Chess, Escuela del Mar, Spain, women.

\section{INTRODUCCIÓN}

En las últimas décadas se han puesto en marcha numerosas políticas orientadas a fomentar la igualdad de género en la educación superior y en la investigación académica, tanto en los países del ámbito Anglo-Sajón, como en los países más avanzados del Centro, el Norte y el Sur de Europa (Addis et al., 2012; Müller et al., 
2011; Rees, 2002). La Estrategia de Lisboa (Consejo Europeo, 2000) y el Programa Horizonte 2020 de la Comisión Europea han reforzado estas iniciativas al urgir a los Estados Miembros a impulsar la igualdad de género en la investigación como herramienta para atraer el mejor talento investigador y convertir la Unión Europea en la economía del conocimiento más dinámica y competitiva del mundo.

Uno de los primeros personajes en Occidente que equipara la mujer con el hombre fue Platón. El filósofo de La República opina que la "diferencia de sexos con todos sus resultados entre los hombres y las mujeres no tiene ninguna importancia, si se demuestra que sus aptitudes y sus cualidades naturales son las mismas en todo lo relativo a las funciones del Estado" (Platón, 1979: 27). Desarrollando este postulado, Platón concluye que hay que dar la misma educación a ambos sexos. De todas formas, el pensamiento del filósofo no se materializó, lo que implicó, con la complicidad de la misoginia de Aristóteles -la mujer es la causa de todos los males-, un sometimiento femenino, tanto en Grecia como a lo largo de los sucesivos años en la sociedad occidental.

Actualmente, aunque se haya avanzado en lo que respecta a la función social y derechos de las mujeres, las desigualdades siguen aún estando presentes. Y aunque las diferencias biológicas sean inevitables, las desigualdades son causadas, en su mayor parte, por el condicionamiento social, que se concreta en la subordinación respecto al hombre, originada, entre otros aspectos, por el papel femenino de "ama de casa", sin capacidad productiva y dependiente económicamente (Beauvoir, 1981; Nash, 1983).

Uno de los hechos principales que relegó y minusvaloró, aún más, a la mujer respecto al hombre, fue, a partir del s. XVII, la "disolución de la família basada en la producción a escala doméstica” (Elschenbroich, 1979: 111). La mujer fue relegada de los conocimientos y se limitó a cumplir las funciones relacionadas con ser madre, con el matrimonio, y con todo un conjunto de tareas serviciales (Álvarez-Uría, Varela, 2009; Avia, 2013; Varela, 2011). En esta línea, en el s. XVIII, Rousseau fue una de las figuras más relevantes a favor de legitimar la subordinación de la mujer respecto al hombre (Calderón, 2005). Pero en este siglo de las luces, aparecen voces que promueven la igualdad de sexos, como Condorcet, Diderot, Campomanes o Feijoo. También, sobresale Josefa Amar y Borbón (1749-1833), que propone una igualdad en los diferentes ámbitos sociales -esto sí, manteniendo el papel femenino de la mujer como esposa y madre- (Amar, 1786, 1790; Feijoo 1778; Huguet, 1989; VicentePedraz, Brozas-Polo, 2014).

Entre otros aspectos, la instrucción será un método para intentar paliar estas desigualdades de género -las luchas de género en este sentido van del brazo de las diferencias de clases sociales-. Pero, aunque se teorice sobre una instrucción de todas las personas, sin distinción de sexo ni nivel económico, nunca se pone en duda ni la supremacía del hombre, ni la de las clases burguesas, y siempre aparecen figuras que discuten esta igualdad (Blanco, 1906; Torné, 2002; Torrebadella, 2011). En palabras de Lerena sobre estas voces iniciales que proponían la instrucción de la mujer: "sí a la instrucción, pero a condición de no discutir o poner en peligro su situación de sometimiento" (Lerena, 1983:185). Encontramos, por lo tanto, durante el siglo XIX que "la ideología de la domesticidad va a configurar un modelo social de familia" 
(Ramos, 2003: 19-32).

El hecho que iniciaría el camino hacia la igualdad, es el cambio social que supuso la Revolución Industrial, que implicó la salida de la mujer de su hogar, además de la posibilidad de ganar un sueldo con el trabajo en las fábricas -eso sí, este sueldo, mucho más bajo que el del varón-. De este modo el sexo femenino, con la posibilidad de producción, gana independencia, y es este el punto de inicio de los esfuerzos para acercar estas diferencias sociales. Estos hechos son claves, como explica Beauvoir, que analiza el papel de la mujer en la sociedad y, ya detalla, que "la opresión social que sufre es consecuencia de su opresión económica” (Beauvoir, 1981: 62). Y como consecuencia cita que: "La emancipación de la mujer no es posible sino cuando esta puede tomar parte en vasta escala en la producción social, y el trabajo doméstico no le ocupe sino un tiempo insignificante” (Beauvoir, 1981: 62).

Partiendo pues de este contexto social, el presente estudio trata la participación de la mujer en las actividades de ocio en la sociedad contemporánea, y se centra específicamente en el ajedrez ${ }^{1}$. Por lo tanto, el objeto de estudio, pretende analizar los inicios ajedrecísticos femeninos en España (1922-1935), reflejo de unos esfuerzos sostenidos para conseguir una mayor equidad e igualdad social de género.

Para tratar el tema, se han consultado fuentes primarias, lo que ha derivado en un análisis hermenéutico de los textos. La búsqueda se ha realizado en las principales bases de datos españolas. Se destaca la hemeroteca digital de la Biblioteca Nacional de España; la Biblioteca Virtual de Prensa Histórica, del Ministerio de Educación Cultura y Deporte del Gobierno de España, y la base de datos del portal Arxiu de Revistes Catalanes Antigues, impulsado por la Biblioteca de Cataluña. Hay además que destacar dos revistas analizadas, aparecidas en los inicios del siglo XX como son Gran Vida (1903-1929) y Feminal (1907-1917). De estas dos publicaciones se han revisado las informaciones relacionadas con la mujer y el deporte. A la vez, y por lo que respecta al ámbito ajedrecístico, se han examinado las principales revistas especializadas de la época: Revista Internacional de Ajedrez (1895-1896), Els Escacs a Catalunya (19271938), Ajedrez. Revista Mensual (1929-1930), Ajedrez Español (1934-1936) (Brasó, 2014b). A esta documentación le han acompañado un conjunto de fuentes secundarias consistentes en tratados, investigaciones y obras que reflexionan sobre la participación social de la mujer, y que han permitido contextualizar estos inicios de la práctica ajedrecística femenina en España, que fijamos alrededor de los años veinte del pasado siglo.

${ }^{1}$ En este artículo el ajedrez será tratado como un deporte más. Es evidente que este argumento causa controversia y tiene sus detractores. Si por deporte nos referimos a la lógica interna de la práctica y al conjunto de actividades que requieren una acción motriz (Lagardera, Lavega, 2003; Parlebas, 2001), no cumple con este requisito. Pero, desde diferentes campos, que no limitan el concepto de deporte a una práctica motriz, se entiende y se justifica el ajedrez dentro del ámbito deportivo (Brasó, 2010; Lagardera, 1999; Santoro, 2010; Termcat, 2010). Para el estudio se ha tenido en cuenta esta segunda perspectiva -más social, cultural y referente a la lógica externa, que es en definitiva el marco de referencia del artículo-. Además, hay que añadir la consideración deportiva del ajedrez según el Comité Olímpico Internacional. 


\section{INICIOS DE LOS MOVIMIENTOS PARA LA IGUALDAD DE GÉNERO EN ESPAÑA}

En España, la Revolución Industrial llega tarde, en relación con países como Inglaterra, Francia o Bélgica, hecho que determina el retraso también en numerosos aspectos culturales donde interviene la mujer. Además, estos inicios no se dan ni en el mismo tiempo ni con la misma intensidad dentro de España. Las zonas periféricas y de fácil acceso al mar, como Cataluña o el País Vasco, fueron mucho más dinámicas también en lo que refiere a la situación de la mujer-, que las regiones interiores. Estas zonas más modernas resultaron pioneras, creando, por lo tanto, grandes desequilibrios en relación con las zonas no periféricas, con un ritmo industrializador más tardío y lento (Iñigo, 2012; Nadal, 1975; Vilar, 1990).

Los movimientos feministas en España tienen sus inicios en los inicios europeos y americanos. Como punto de inicio destaca La Declaración de los derechos de la mujer y la ciudadanía (1791) -redactada por Olympe de Gouges- y dónde se propone una igualdad jurídica y legal entre hombres y mujeres. Posteriormente hay que citar la obra de Mary Wollstonecraft (1792), que reclama esta igualdad entre seres humanos. Durante el s. XIX surgen voces como la de Jeremy Bentham o John Stuart Mill, que postulan que una felicidad de la sociedad sólo puede conseguirse con la libertad del ser humano, también de la mujer. En este ámbito extranjero, destaca también la Declaración de Séneca Falls (1848) -en Nueva York-, en favor de un trato igualitario en el ámbito legal. Además, los análisis de Bebel, Marx, Engels o Robin entre otros, son de suma relevancia y críticos con estas desigualdades femeninas. Equiparan la dominación del hombre con la lucha de clases, y como consecuencia, hacen frente al capitalismo para conseguir la libertad de la mujer (Bebel, 1977; Engels, 1996; Gouges, 1989; Marx, Engels, 1981, 2012; McMillen, 2008; Robin, 1981; Sánchez, 1982; Stuart, 2008). En España, diferentes voces de la época empiezan a analizar las desigualdades en lo que respecta a los derechos de la mujer. Los pensamientos de Flora Tristán (1803-1844) marcan los inicios feministas socialistas, juntamente con figuras como Clara Campoamor (1888-1972), Concepción Arenal (1820-1893) o María de la Concepción Gimeno de Flaquer (1850-1919) (Arenal, 1974; Ayala, 2009; Campoamor, 1936; Tristan, 1844).

Además, analizando las trayectorias de determinadas mujeres del s. XIX, este ideal igualitario es un reflejo de las conexiones entre republicanismo, laicismo y feminismo (Ramos, 1999). Se crean y aparecen, dentro y fuera de España, asociaciones, escuelas, ligas, congresos, folletos y prensa feminista (Ramos, 2003). Un ejemplo es el caso de las fourieristas gaditanas y sus reivindicaciones que plasmaron en El Pensil Gaditano (1856). Relacionado con este feminismo del movimiento librepensador es destacable la figura de Rosario de Acuña (1851-1923).

Había, así, una estrecha relación con el regeneracionismo español de finales del s. XIX. La expansión de las ideas de Acuña dio lugar, en 1889 a la Sociedad Autónoma de Mujeres de Barcelona, fundada por Ángeles López de Ayala, Teresa Claramunt y Amalia Rodríguez. Le siguieron otras como: la Asociación General Femenina de Valencia (1897), la Sociedad Progresiva de Barcelona (1898) -conversión de la 
agrupación de 1889-, la Federación Malagueña de Sociedades de Resistencia (1897), la Unión Femenina de librepensamiento de Huelva (1897), la Sociedad de Mujeres Librepensadoras de Mahón (1899) y la Sociedad Progresiva Femenina de Málaga (1900) (Cortada, 2008; Moral, 2007; Ramos, 1999; Sanfeliu, 2008). Es destacable el seguimiento de estas ideas, como por ejemplo en las manifestaciones barcelonesas en junio y julio de 1910 por parte de la Sociedad Progresiva Femenina, y por López de Ayala (Arce, 2007).

Una representación de la concienciación social en favor de las ideas feministas se encuentra en la petición del derecho a voto, a partir de 1919, y su reconocimiento en 1931, hecho que muestra, después de un duro camino, la voluntad por conseguir la igualdad (Sanfeliu, 2008).

Estos avances también se dan en el ámbito educativo, a partir del s. XX, con ideas que empiezan a promover la coeducación (Serrano, 1992), aunque los inicios en favor de la igualdad hay que buscarlos en la Ley Moyano (1857)-, que dictaminó la obligatoriedad de educación de niños y niñas -entre 6 y 9 años-. Esto implicaba la creación de escuelas, también para maestras (Ajuntament de Barcelona, 1922; Lerena, 1976). Cabe destacar la Institución Libre de Enseñanza (1876), encabezada por Giner de los Ríos (1839-1915) que tiene en su ideal la educación de la mujer. También es remarcable la figura de Pedro de Alcántara García (1842-1906), que abogaba por un pueblo culto mediante la educación de todas las esferas sociales, incluyendo una educación de la masa popular, es decir, las clases bajas y las mujeres (García, 1879; Martín, 1989). Pero, aunque se progresó, el currículum femenino iba básicamente destinado a las labores del hogar, y estas voces del último tercio del s. XIX que reclaman mayores derechos para la mujer son poco significativas. No es hasta el s. XX que estas ideas se materializan, gracias principalmente a las leyes de 1901, 1909 y 1910, que van reduciendo las diferencias de género (Amo, 2009; Cortada, 2008; Moreno, 2009). Una figura destacable es la del pedagogo Ferrer y Guardia que propone una educación interclasista y sin barreras de género (Delgado, 1981; Ferrer, 1990).

Estos progresos igualitarios son un reflejo sobre la consideración de la mujer, y se hacen también tangibles en el ámbito deportivo, que es, "uno de los principales cotos masculinos" (Elias, Dunning, 1986: 324). Los ideales masculinos y femeninos, en este campo son más que evidentes, mostrando un ideal masculino relacionado con la vigorosidad y la fuerza, y otro de femenino, débil, dependiente y temeroso (García, Flores, 2008).

Como consecuencia, la participación de la mujer en los juegos y deportes en todos los periodos no deja de ser un reflejo más de su papel y de la división social existente. Se comprueba que los juegos bélicos y de alta actividad física se recomiendan para niños, y los calmados y relacionados con el hogar, con el sexo femenino (Amar, Jauffret, 1807; Bastinos, 1896; Caro, 1884; Fernández, 1852; Fernández, 1864; Marco, Ochoa, 1895; Naharro, 1818; Rementería, 1839; Santos, 1876). Así, los valores masculinos relacionados con la agresividad y la fuerza conducen a un dominio generalizado de los hombres sobre las mujeres (Elias, Dunning, 1992).

Un hecho destacable es la modernización de los juegos violentos en Gran Bretaña 
a inicios del s. XIX, con el objetivo de restringir la violencia, que juntamente con la creciente y similar civilización en el ámbito deportivo, fueron causas que redujeron las desigualdades, en estos sectores (Elias, Dunning, 1992). Estos sucesos, que van ligados a la industrialización británica, permitieron un mayor protagonismo de la mujer que buscaba una liberación y emancipación, y se oponía a las voces de la época contrarias a esta práctica femenina. Estos hechos se plasman con los primeros -y testimonialesclubes y asociaciones femeninas deportivas a finales del s. XIX y su incremento en torno a 1920. De todas formas, como sigue sucediendo a día de hoy, las desigualdades y el dominio deportivo masculino son evidentes (Puig, Soler, 2004; Pujadas, 2010, 2011; Torrebadella, 2016). Un reflejo de todo ello se ve, En España, con la práctica minoritaria deportiva a finales del s. XIX y hasta los inicios del XX. La Revolución Industrial y las influencias inglesas ayudan a originar estos movimientos. Simón detalla que estos principios son promovidos por ciudadanos ingleses y escoceses residentes en España (Simón, 2009). A éstos, se les unirán los hijos de las familias de la elite social, muchos de ellos con influencia inglesa, por el hecho de haber cursado sus estudios en las public schools británicas (Carbajosa, Riaño, 2009; Sánchez, Rivero, 2013; Simón, 2009; Torrebadella, 2011, 2013).

De todas formas, con la Revolución Industrial, las largas jornadas laborales y los bajos recursos económicos de los trabajadores en las fábricas, hay un impedimento para el ocio y la práctica deportiva, excepto para el estamento de las clases altas. A este aspecto hay que sumarle la masculinidad en las actividades de tiempo libre -como fue la práctica del ajedrez, que tenía lugar en salones, clubes y cafés (Brasó, 2014b), y en la que no aparece la mujer burguesa hasta los años treinta del s. XX-.

En el ámbito femenino, destaca el caso de la Casa Real. Además de Alfonso XIII, destacan las figuras de la Infanta Isabel -que montaba a caballo, practicaba la caza y era aficionada a los toros (García, 2008; Ortega, 1943) - y la reina Victoria Eugenia que impuso una renovación de la mujer en la Corte y potenció la práctica del tenis, el golf y la equitación (Gómez-Santos, 1993)-. A esta participación hay que añadir otro hecho destacable beneficioso para la incorporación de la mujer en la sociedad y el deporte: el movimiento regeneracionista de inicios del s. XX. Así, las reivindicaciones femeninas por el derecho al ocio y al deporte fueron significativas, aunque tuvieron que enfrentarse a argumentos que abogaban por un ideal tradicionalista de mujer, asociado a las funciones del hogar.

El discurso regeneracionista pretenderá fomentar la salud, también, de la mujer, esto sí, manteniendo su función maternal y su feminidad. Como consecuencia, el deporte se irá asociando a un concepto de modernidad, además de convertirse en un "medio ideal para favorecer las relaciones entre los grupos más privilegiados” (Simón, 2009: 80).

A partir de los años veinte del siglo pasado, Barcelona, encabezó este proceso de deportivización de la mujer con la práctica de diferentes mujeres pioneras que formaban parte de clubes, asociaciones y entidades para la práctica. Estas iniciativas tuvieron su máxima repercusión a partir de 1931, con la Segunda República -que propició mejoras laborales; mayor tiempo libre; una ley del matrimonio civil y del divorcio; el derecho de voto de la mujer y poder ser elegidas en cargos públicos; la 
abolición de la prostitución; la baja por maternidad; la legislación del aborto en Cataluña... - . Es a partir de esta época cuando se incrementa la participación femenina en el deporte, gracias también al ascenso de una popular la clase media (Pujadas, 2011). No obstante, la guerra civil acabará de cuajo con este incipiente proceso que tuvo lugar en la Segunda República, y que había avanzado en lo que se refiere a la liberación y equidad social de la mujer.

Para ejemplificar estos inicios, se han tratado dos publicaciones periódicas de referencia. La primera es Gran Vida (1903-1929), donde se visualiza la influencia inglesa en lo que respecta a la participación deportiva femenina, y sus inicios en España (Sánchez, Rivero, 2013). También se verifica que hay muestras de desigualdad y reticencias por la participación de la mujer en determinadas actividades. Hay que remarcar que algunos artículos, los firma el mismo director de la revista: Vicente de Castro Les (A propósito del gran..., 1922; Alzamora, 1910, 1914a, 1914b, C. L., 1918, Castro, 1914, 1915; Dalbanne, 1913; Deportes de nieve, 1923; Deportes femeninos, 1921; El concurso hípico de Madrid, 1927; Intervención de las mujeres..., 1906; La aptitud de la mujer..., 1927; Los deportes apropiados para..., 1922; Los deportes en la juventud..., 1915; Los deportes en Santander..., 1918; Los deportes y el feminismo, 1925). Otro ejemplo, de estos esfuerzos por romper la concepción tradicionalista de la mujer y potenciar el feminismo, se encuentra en la revista catalana Feminal (19071917). La publicación dirigida por Carme Karr, es un suplemento mensual de La Ilustració Catalana. La práctica deportiva femenina catalana se muestra en la revista, para hacer frente al sedentarismo, pero también para promover los deportes, carentes de aficionadas en el país, a diferencia de la práctica de casi todos los deportes en el extranjero por parte de las mujeres. Ya desde sus primeros números, se tratan deportes, clubs, asociaciones deportivas y mujeres relacionadas con el tenis, la hípica, el automovilismo, los globos, la pesca, el tiro, el tiro con arco, el esquí o el excursionismo (B. M., 1908; Bouyer-Karr, 1907; Casademunt, 1908; Concurs de globos..., 1908; E., 1907a, 1907b, 1907c, 1908a, 1908b; F. 1907, 1908; Fransa, 1908; Karr, 1907; L’hivern de 1908 en..., 1908).

Analizando estas publicaciones que versan sobre la situación social de la mujer en los inicios de los años veinte y la práctica deportiva femenina, se comprueba que el ajedrez femenino en España aparece con retraso en relación con otros países de Europa.

\section{EL AJEDREZ Y LA MUJER EN EUROPA}

Analizando estas publicaciones que versan sobre la situación social de la mujer en los inicios de los años veinte y la práctica deportiva femenina, se comprueba que el ajedrez femenino en España aparece con retraso en relación con otros países de Europa.

El ajedrez se ha relacionado con la guerra, la vida, y también con la mujer. Y en relación con este último aspecto, el sexo femenino es utilizado muchas veces como un objeto puramente material. De este modo, en numerosas ocasiones, ha sido elemento de disputas, hecho que muestra la dominación masculina de la sociedad. Hay, pero, que 
detallar que se encuentran algunos relatos ajedrecísticos relacionados con la fuerza y el valor femenino en la Edad Media (Brunet, 1891; Pareja, 1935; Vinyoles, Martín, Chalaux, 2003). En este punto, hay que tratar el poema Scachs d'amor, y posteriormente a Cesolis y Vicent. En sus respectivas obras, dan un valor significativo a la mujer y se le concede a la dama de ajedrez una gran amplitud de movimientos, simbolizando el poder de algunas reinas de la alta Edad Media y, concretamente, el poder de Isabel La Católica (Pelaz, 2011; Wade, 2003). Diferentes investigadores han considerado la figura de la reina española, a finales del s. XV, como clave para el paso del ajedrez antiguo al moderno, así como para la creación del juego de las damas (Garzón, 2010; Westerveld, Garzón, 2004).

Analizando la obra de Alfonso $\mathrm{X}$, se encuentran numerosos grabados en los cuales la mujer juega al ajedrez. Este hecho remarca la fuerza que adquirió el sexo femenino, y permite entender el estorbo que significó y la voluntad a menudo por apartar la mujer del poder y la política. Sea como sea, la sociedad, era masculina y "la reina puede tener autoridad, pero no poder" (Pelaz, 2011: 186).

Por lo que respecta a los inicios de la práctica social ajedrecística femenina, se podría citar el caso de la aldea de Storbeck (Meyer, 1930; Paluzíe, 1921; Storbeck, la ciudad..., 1851). La excepcionalidad de la localidad, donde todo el mundo es practicante, permite fijar uno de estos orígenes. De todas formas, la práctica masiva de la mujer en el juego no se produce hasta el último tercio del s. XIX en Inglaterra y Francia.

En este período tenemos constancia en la prensa española de informaciones que, por su excepcionalidad, anuncian la práctica ajedrecística del sexo femenino. A mediados del s. XIX, en El Museo Universal, se cita que, en relación con el ajedrez, "son muchas las madamoisselles y ladys que lo acogen con la predilección que se merece” (Martínez, 1865: 199). Se comprueba que la participación femenina, habitual en países como Francia, Inglaterra o América, es un hecho excepcional en España. Es destacable también la noticia aparecida en el Diario de Gerona: "Una americana, la señora W. J. Boird, es designada con el nombre de Reina del Ajedrez, a causa de su habilidad superior en este difícil juego. Lo más curioso es que su hija de edad de trece años, presenta en el más alto grado las mismas aptitudes.” (Curiosidades, 1894: 14)

Asimismo, a finales del s. XIX ya hay constancia de clubes de jugadoras en Londres, como el Ladies' College Chess Club, creado en torno a 1878. La Revista Internacional de Ajedrez destaca también el Ladies Chess Club, fundado en 1895 y presidido inicialmente por la Sra. Newnes. Los inicios del club son obra de veinticinco mujeres, aunque un año más tarde ya tenía noventa y cinco socias, y en 1906, más de 125. Entre las actividades y visitas de diferentes figuras ajedrecistas destacan enfrentamientos, destacando el de veinticinco tableros, con los ajedrecistas del Metropolitan Chess Club, el 13 de marzo de 1896 (Henry, 2015; Noticias, 1896). Estos hechos muestran las inquietudes por la práctica y el asociacionismo ajedrecístico.

Un año más tarde, se organiza, también en Inglaterra, el primer campeonato internacional femenino del que hay constancia, con veinte participantes (El ajedrez femenino, 1932). Posteriormente ha habido torneos internacionales, como los de Merán 
(1924) o Viena (1924 y 1926). Y es en los años veinte, con la ajedrecista Menchik (1924), que la mujer empieza a combatir con el hombre en iguales condiciones. La jugadora -que también jugaba a damas-, incluso en el torneo de Ramsgate se clasificó a muy pocos puntos del vencedor Capablanca y pasó por delante a jugadores como Rubinstein (El ajedrez femenino, 1932).

Es relevante añadir además de la figura de Menchik, a Graf o Semmler, cuyos enfrentamientos de alta calidad ajedrecística tuvieron un gran impacto internacional (Graf, 1935; Sección de partidas, 1936; Menchick, 1935).

La publicación valenciana Ajedrez, también publicó noticias y partidas relacionadas con el ajedrez femenino. La mayoría de las informaciones hacen referencia a la campeona mundial Menchick, aunque aparecen datos de menor relevancia de otras jugadoras (Campeonato Mundial Femenino, 1930; Información extranjera, 1930; Partida núm. 17..., 1930; Tartakower, 1929, 1930). Por lo tanto, la aparición de la mujer va cogiendo fuerza gradualmente. Tartakower reproduce las palabras de Menchik sobre esta necesidad en la aparición de la mujer en el juego: "El porvenir del ajedrez exige la cooperación de la mujer” (Tartakower, 1930: 75).

Así, se puede constatar que en los años veinte y treinta, el ajedrez femenino coge ímpetu. Junto con las primeras ajedrecistas de repercusión internacional, aparecen diferentes eventos femeninos. Las noticias sobre enfrentamientos con una alta repercusión y participación, por ejemplo, en Francia son frecuentes (Madame d'Autremon..., 1932; Partida 883, 1932; Partida 892. Campionat Femení de França, 1932; Vidal, 1926). Guinart (1933) ya detalla la falta de participación española en estas partidas internacionales, y más teniendo en cuenta que en este período ya se están organizando torneos femeninos en diferentes poblaciones de Cataluña.

Estos inicios del ajedrez femenino aparecidos en Europa, y que tienen en Francia una significación relevante, aparecen con retraso en España, alrededor de los años treinta. $^{2}$

\section{EL AJEDREZ FEMENINO EN ESPAÑA}

En España, durante los años veinte se empiezan a localizar noticias que tratan la práctica de la mujer y el ajedrez, aunque su consolidación no aparece hasta una década después. Esta participación se relaciona a la vez con la práctica del juego en los jóvenes, ya sea en la escuela, las universidades o en los clubes (Sánchez-Ocaña, 1935).

Uno de los movimientos iniciales ajedrecísticos femeninos puede fecharse con el movimiento de la Escuela Nueva, en 1922. En este año, Pedro Vergés promueve -en la Escuela del Mar de Barcelona y en las colonias a Vilamar en Calafell- un modelo coeducativo centrado en la vida social y los juegos, con protagonismo del ajedrez (Vergés, 1933). Estos centros -con un modelo similar en la actualidad y gran protagonismo del juego (Brasó, 2014a, 2014b; 2015; Torrebadella y Brasó, 2014)- son

\footnotetext{
${ }^{2}$ Este retraso también se comprueba con relación a Argentina, en la cual ya se dedica parte de un libro a tratar el ajedrez y la mujer (Pérez, 1920). 
los iniciadores del ajedrez femenino en la juventud. Cabe decir que este hecho se constata en las explicaciones del pedagogo en Els Escacs a Catalunya, concretando que la Escuela del Mar fue la pionera de esta práctica en las escuelas de Barcelona.

En el ámbito asociativo, uno de los pioneros en promover el ajedrez femenino fue el Club Ajedrez de Barcelona, impulsor de la Federación Catalana de Ajedrez y posteriormente de la española. A finales de los años veinte, el Club Ajedrez de Barcelona -creado en 1921- tiene una sección femenina numerosa. De todas formas, aunque se demuestra esta afición ajedrecística femenina, en determinados comentarios aparece el machismo de la época: “A aquellos desgraciados maridos que disfrutan de esas señoras insufriblemente charlatanas les recomiendo que procuren aficionarlas al ajedrez. Es un curioso descubrimiento que no sé si llegará a ser sensacional: jugando al ajedrez ¡las mujeres no hablan!” (Sánchez-Ocaña, 1935: 14).

Son destacables también las exhibiciones simultáneas de ajedrez femeninas en 1925 llevadas a cabo por el club barcelonense Ruy López. En el evento, hubo numerosa expectación, hecho que permite inferir la importancia que empieza a dársele al juego. Muchos personajes de prestigio acuden a la cita, como el campeón de Cataluña, en 1924, Plácido Soler, los ajedrecistas Rafael Doménech y José Vilardebó, socios de la Peña Graciense, personalidades del Club de Ajedrez Barcelona, o figuras del Escacs Comtal Club, además de la totalidad del comité de la futura Federación Catalana de Ajedrez, la junta directiva del Club Ruy López, y socios, mujeres y multitud de personas (Notas locales, 1925). En el evento, el director del Club Ruy López explicó "los proyectos que tiene la entidad, con respecto a la formación de su sección femenina y animando a las señoras y señoritas presentes a que ingresen en sus filas para cooperar al desarrollo del ajedrez entre el sexo femenino" (Notas locales, 1925: 31). Participaron nueve jugadoras, de las cuales destaca una niña de seis años. El bajo número de jugadores se justifica por el hecho que las simultáneas habían sido anteriormente aplazadas, y muchas ajedrecistas no pudieron acudir a la nueva llamada. De todas formas, el éxito del evento ya hacía pensar a los directivos en la organización de otras celebraciones. Posteriormente aparecen otros eventos como el torneo nacional de 1926, las tablas de la señora Benet con el que fue campeón de Cataluña en 1933, Ángel Ribera en unas simultáneas -en 1930-, o la partida entre el señor Soler i la señora Mechik en el torneo barcelonense de 1929 (Ajedrez. Torneo Nacional..., 1926; De toda España, 1930; Partida. Variante Primera, 1930).

Pero no es hasta la década de los treinta que surge realmente un creciente interés por el ajedrez femenino. Además de la organización de diferentes torneos, cabe incidir en el Dr. Rey Ardid, campeón de España. El ajedrecista, además de narrar los múltiples beneficios del juego para hombres y niños, trata también esta práctica en la mujer, en el periódico La Vanguardia. Explica la poca práctica femenina en el S.XIX en contraposición a la alta participación y el alto nivel ajedrecístico femenino de la época a causa de la incorporación progresiva de la mujer en las actividades humanas. Piensa además que el ajedrez se adapta a la psicología femenina:

“... ya que la astucia y la tenacidad, dos facultades que posee la mujer quizá en mayor grado que el hombre, son la base de la lucha que contribuye una partida de ajedrez [...] la fina sensibilidad de la mujer le sabrá presentir los peligros con 
mayor antelación y le ayudará a tender las celadas más sutiles y peligrosas.” (Rey, 1932: 10)

Estos inicios se consolidan en la década de los años treinta. Muestra de ello es el Campeonato Infantil Individual de 1932. En el torneo ya aparecen numerosas niñas ajedrecistas como Martínez, Argimon o Santasusagna. Además, personalidades dentro de las entidades de ajedrez incitan a la práctica femenina (Santasusagna, 1932).

El mismo Dr. Rey trata posteriormente otra vez el tema ajedrecístico en la mujer, y entre otras cosas, felicita al Club de Ajedrez Barcelona por la iniciativa de la organización de un torneo femenino para fomentar la práctica del juego en este grupo de población. Incluso la noticia llega a Madrid y, en El Sol se comenta el hecho que estos eventos son uno de los mejores medios de propaganda para la práctica femenina. Y del 18 de diciembre de 1932 al 12 de marzo de 1933 se juega el primer torneo femenino de ajedrez en España. El evento se realizó en la sede del local organizador en la Rambla Cataluña, 4. El éxito fue notorio, con alta participación, multitud de espectadores y gran repercusión mediática (Blanco, 2006; Rey, 1933; Sagarra, 1932). Además, entre los asistentes destacaban los presidentes del Club de Ajedrez de Barcelona, de la Federación Española -señor Marín-, de la Federación Catalana señor Llorens-, delegados de las regiones participantes, y muchas otras personalidades (Blanco, 2006; Guinart, 1933; Puig, 1933; Sanz, 1933a, 1933b). Este torneo, ganado por la señora Zengotita, desencadenó en la creación de la Sección Femenina del Club de Ajedrez Barcelona, en 1932, compuesto por veinte mujeres, entre las cuales había las socias del Club d'Escacs Mollet, Marta Puigcercós y Winie Ortega ${ }^{3}$. Dichas jugadoras ya obtuvieron anteriormente el $1^{\circ}$ y $2^{\circ}$ lugar, en la categoría $C$ del segundo torneo social, del citado club, hecho que significó el ascenso de una categoría en la siguiente edición del torneo (Blanco, 2006).

En estos años aparecen diferentes equipos y secciones femeninas de ajedrez, con una alta actividad. Un ejemplo de estos esfuerzos a favor de la práctica de la mujer es el homenaje a la sección femenina de ajedrez, en San Feliu de Llobregat, mediante una sesión de simultáneas proporcionada por el campeón de Cataluña Plácido Soler (De información general..., 1933). También puede visualizarse la actividad de la sección femenina, del Club de Ajedrez Barcelona, en la Fiesta del Ajedrez de Manresa, el 2 de abril de 1933. Y también son relevantes las simultáneas que el jugador del Club de Ajedrez de Barcelona, Rafael Domènech ofreció a la Peña de Ajedrez de Mollet el 14 de abril de 1933. Es notorio destacar que la única partida que perdió fue contra la ajedrecista Puigcercós (Blanco, 2006).

También destacable y que muestra los progresos femeninos del ajedrez en la década de los años treinta, es la conferencia de la señora María Luz Morales en las fiestas del doceavo aniversario del Club de Ajedrez de Barcelona. La ponencia, titulada La mujer y el ajedrez, fue organizada por la sección femenina de esta entidad (Morales,

${ }^{3}$ Ocuparon el segundo y tercer lugar en el Primer Campeonato Femenino celebrado a Cataluña, de 1932. En las siguientes ediciones -1934 y 1935-, Winie Ortega ocupó la segunda y tercera posición respectivamente. Puigcercós, en cambio, no participó. Zengotita ganará también la siguiente edición del torneo, de 1933. 
1933).

Posteriormente, es pertinente subrayar el enfrentamiento de Puigcercós, el 1936, con la segunda mejor jugadora del mundo: Sonia Graf. La Peña de Ajedrez de Mollet se encargó de organizar el evento, que finalizó con el resultado de 3 a 1 a favor de Graf. Además, al cabo de unos días, y también organizado por la misma entidad, Graf dio unas simultáneas cediendo únicamente una partida. En este año 1936, fue notoria también la participación de Puigcercós, Velat y Ruiz en el torneo mayor internacional

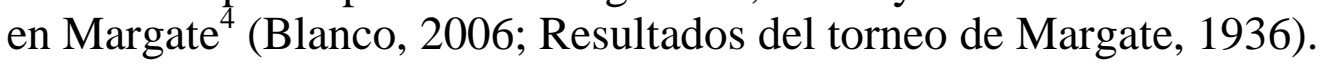

Por lo que respecta a Madrid, en el Casino militar se lleva a cabo un torneo femenino de relevancia, organizado por la Sección de Ajedrez del Centro del Ejército y la Armada, dos años después -1934- que el organizado en Barcelona (Rey, 1934a; García, 1934; Sanz, 1934). Es curioso remarcar que se informa que es el primer torneo de estas características, hecho que no es verídico, teniendo en cuenta el torneo del año 1932 en Barcelona. La información en el $A B C$ tampoco da detalles del campeonato barcelonense de unos años anteriores (Golmayo, 1934).

En 1934 el Dr. Rey, que ya habla de la mayor actividad ajedrecística barcelonense en relación con Madrid, propone un encuentro entre ambas ciudades. Así, el encuentro entre la campeona de la ciudad Condal, Zengotita, y la señorita Pérez, campeona del Torneo de Madrid "estimularía a las mujeres a seguir engrosando las filas de la afición española” (Rey, 1934b: 12).

La figura de Sanz, periodista de la Luz y director-fundador de El Ajedrez Español, es relevante en el sentido que va tratando aspectos sobre la mujer y el ajedrez. Además, de informaciones relativas a los torneos femeninos, en 1934, reflexiona sobre el aumento, insólito unos años antes, de la participación de la mujer en los deportes físicos. La justificación a “este próspero desarrollo deportivo” viene dada "por la gran labor de divulgación y proselitismo realizada en simpática camaradería por la masa estudiantil de ambos sexos, que ha actuado con el ardor y la despreocupación propios de la juventud” (Sanz, 1934: 14). Por lo tanto, son los jóvenes de ambos sexos que en su período formativo potencian el ajedrez para igualarlo al nivel de práctica masculino. Justifica su interés diciendo que el ajedrez favorece: "al espíritu y la inteligencia lo que el deporte físico para el organismo [...] ambos ejercicios, el muscular y el mental, se complementan perfectamente en un equilibrio muy conveniente para evitar una excesiva y perjudicial absorción de cualquiera de ellos” (Sanz, 1934: 14) ${ }^{5}$.

Asimismo, el periodista numera otras cualidades de interés para la mujer. De este modo el juego no tiene el materialismo de los juegos de cartas y azar, es óptimo para

${ }^{4}$ Ciudad inglesa de alta tradición ajedrecística, dónde se han llevado a cabo diferentes congresos y torneos internacionales. El del año 1939 fue incluso publicado en forma de libro (De Agustín, 1944).

${ }^{5}$ Es remarcable detallar que se entiende la práctica deportiva como la mera ejercitación muscular, y el ser humano como hombre-máquina. Lógicamente es un texto de la época y es evidente que en la actualidad se consideran básicos para la práctica deportiva inteligencias como la motriz, la emocional o la perceptiva (Damasio, 2001, 2005, 2010; Goleman, 1996). 
todo el mundo, enseña a razonar, proporciona enseñanza muy rápidamente, además de relacionarse con los conceptos femeninos de sensibilidad, gusto artístico, astucia, economía o prudencia. También detalla el estado de la cuestión y reflexiona sobre la práctica deportiva femenina (La Redacción, 1935). Además, destaca la aparición femenina en la sociedad y especialmente en los deportes y el ajedrez:

“donde más resalta la invasión femenil es en los deportes físicos: los gimnasios, campos de atletismo, fútbol, hockey, basket-ball, las piscinas, se encuentran materialmente dominadas por la mujer [...] los varones ven estupefactos acercarse el fin de su hegemonía deportiva.

[...] esta ola feminista no podía detenerse en el ajedrez, juego que, aunque hasta ahora ha parecido ser patrimonio de "sesudos homes", precisamente por esto, por su prestigio intelectual y por sus dificultades, ha excitado más en la mujer el deseo de conocerlo y dominarlo, pues su actual impulso y afán de conquista no conoce límites. Su especial sensibilidad aporta nueva savia al juego, y su presencia en los salones de ajedrez, embelleciéndolos, es un nuevo aliciente para los ajedrecistas.” (La Redacción, 1935: 369) ${ }^{6}$.

Así, en España en la década de los años treinta, se va incrementando la participación de la mujer en el ajedrez -e incluso se lleguen a narrar partidas femeninas que no se refieren a ningún torneo, que tienen un carácter amistoso, y que por lo tanto gozan de poca relevancia- (Matx Velat-Puigcercós, 1935). Y encabezando este movimiento, hay que nombrar la ciudad de Barcelona que como ya se ha comentado juega un papel clave en el inicio de la práctica femenina del ajedrez. Sánchez-Ocaña (1935) describe de este modo la supremacía de Barcelona en el ámbito del ajedrez femenino con "cuarenta clubs federados y una multitud de peñas y sociedades pequeñas sin federar. Hay veinte mil jugadores de todas las categorías y supporters o hinchas, y equipos invencibles. Allí juegan al ajedrez las chicas solteras -hasta que se casan, naturalmente-; las damas provectas, las niñas, los niños...” (Sánchez-Ocaña, 1935).

Como colofón, hay que decir que todas estas iniciativas y progreso del ajedrez femenino eran un reflejo de la liberación y la equidad de la mujer. De todas formas, los avances que se habían conseguido, principalmente durante la II República y como sucedió con muchos otros ámbitos sociales y culturales quedaron paralizados a causa de la Guerra Civil Española (1936-1939) y la posterior dictadura franquista.

\section{A MODO DE CONCLUSIÓN}

La tardanza de la llegada de la Revolución Industrial en España marcó un retraso social en lo que respecta a la igualdad de oportunidades de género. Como consecuencia, la práctica femenina del ajedrez y del deporte en general, llega tarde en

${ }^{6}$ El texto es una muestra, a la vez, de la reacción conservadora ante los avances educativos y sociales de la República. Aunque se hable de invasión femenil, hay que remarcar que las prácticas de las mujeres en estos ámbitos eran aun minoritarias. 
relación con Inglaterra, Francia, y en general el resto de Europa.

La emergencia de los movimientos feministas, de finales del s. XIX e inicios del $\mathrm{XX}$ determinaran estos balbuceos ajedrecísticos femeninos. La divulgación en la prensa de los eventos y jugadoras de ajedrez es el preludio de la práctica del juego por parte de la mujer española.

Hay que incidir en la ciudad de Barcelona -en la década de los años veinte-, y la Escuela del Mar del pedagogo Vergés. Su modelo, coeducativo y basado en el uso competitivo de los juegos y el ajedrez, parece ser un punto de partida de esta actividad en la mujer. Se podría vincular la aparición de la práctica ajedrecística femenina con el movimiento de la Escuela Nueva barcelonesa. A partir de este momento, en el ámbito educativo, el ajedrez se empieza a tratar en los colegios, en las colonias, y por parte de clubes y asociaciones. Estas entidades, irán incorporando secciones femeninas, que competirán, alrededor de la década de los treinta, en diferentes eventos.

Se comprueba que durante la década de los años veinte del siglo pasado, empieza a visualizarse la mujer en la práctica ajedrecística, y en los años treinta se van consolidando estos inicios, que se relacionan con las libertades de género que propicia la Segunda República.

En el ámbito asociativo, uno de los iniciadores fue el Club Ruy López de Barcelona, aunque es el Club de Ajedrez Barcelona la entidad que dio más impulso a la práctica femenina. La organización del primer torneo femenino en España, en 1932, es una muestra de ello.

Por lo tanto, es destacable este movimiento, que al fin y al cabo es social. Sanz (1934) comenta que son las masas estudiantiles, hombres y mujeres, los que potencian el juego en la mujer, por lo tanto, se pretende avanzar en la igualdad o, al menos, disminuir las diferencias en las prácticas, como ya sucedía unos años antes con algunos deportes, como el tenis y el excursionismo. Este movimiento ajedrecístico femenino que durante la II República cogió impulso, del mismo modo que pasó con los modelos sociales y educativos renovadores, se paralizó con la Guerra Civil Española.

\section{REFERENCIAS BIBLIOGRÁFICAS}

A propósito del gran Concurso de tenis (1922): Gran Vida, n² 234, 358.

A. C. (7 septiembre 1932): De Ajedrez. El Sol, 7.

Ajedrez. Torneo Nacional de Ajedrez (24 septiembre 1926): El Sol, 6.

AJUNTAMENT DE BARCELONA (1922): Les construccions escolars de Barcelona: recull dels estudis, projectes $i$ altres antecedents que existeixen en l'Ajuntament per a la solució d'aquest problema. Barcelona: Henrich i Ca.

ÁLVAREZ-URÍA, FERNANDO; VARELA, JULIA (2009): Sociología de las instituciones. Madrid: Morata. 
ALZAMORA, ANTONIO (1914a): Deportes atléticos. La marcha y la carrera á pie. Gran Vida, $\mathrm{n}^{\mathrm{0}}$ 138, 342.

ALZAMORA, ANTONIO (1914b): Deportes atléticos. Natación. Gran Vida, n 136 , 278-281.

ALZAMORA, ANTONIO (1910): La cultura física en la mujer. Gran Vida, nº 85, 168.

AMAR, JOSEFA (1786): Discurso en defensa del talento de las mujeres, y de su aptitud para el gobierno, y otros cargos en que se emplean los hombres. Memorial literario, $\mathrm{n}^{\circ}$ 32: 400-430.

AMAR, JOSEFA (1790): Discurso sobre la educación física y moral de las mujeres. Madrid: Imp. Benito Caro.

AMAR, JEAN-AGUSTIN; JAUFFRET, LOUIS-FRANÇOIS (1807): La gimnástica ó escuela de juventud. Madrid: Imp. de Álvarez, 1807.

AMO, MA. CRUZ DEL (2009): La educación de las mujeres en España. De la amiga a la Universidad. CEE Participación Educativa, nº 11, 8-22.

ARCE, REBECA (2007): Dios, patria y hogar: la construcción social de la mujer española por el catolicismo y las derechas en el primer tercio del siglo $X X$. Santander: Ed. Universidad de Cantabria.

ARENAL, CONCEPCIÓN (1974): La Emancipación de la mujer en España. Madrid: Jucar.

AVIA, MARÍA DOLORES (2013): Feminismo y libertad. Comentarios a los textos de Julia Varela y Fernando Álvarez-Uría. Papers: revista de sociologia, nº 98, 647651.

AYALA, MARÍA DE LOS ÁNGELES (2009): Concepción Gimeno de Flaquer. El problema feminista. En Pilar, M. y Rodríguez, M. ed., Vivir al margen: mujer, poder e institución literaria, 291-301. Segovia: Instituto Castellano y Leonés de la Lengua.

B. M. (1908): Una aeronauta catalana a Vigo. Feminal, nº 18, 4.

BASTINOS, JULIÁN (1896): Juegos Infantiles. Barcelona: Lib. de Antonio J. Bastinos ed.

BEAUVOIR, SIMONE (1981): El segundo sexo. Buenos Aires: Aguilar.

BEBEL, AUGUST (1977): La mujer y el socialismo. Madrid: Akal. 
BLANCO, JOAN (2006): 75 aniversari. Club d'Escacs Mollet. Abril 1931-2006. Mollet: Minerva Mollet.

BLANCO, RUFINO (1906): Tratado de pedagogía. Madrid: Revista de Archivos.

BOUYER-KARR, VIOLETTE (1907): Lo que's diu a Fransa. Feminal, nº 5, 12.

BRASÓ, JORDI (2010): Escacs i aprenentatge. Girona: Papers on demand.

BRASÓ, JORDI (2014a): Diseño y validación de un test de ajedrez -T23- para niños de 9 a 12 años. Ágora para la educación física y el deporte, nº 16(1), 18-35.

BRASÓ, JORDI (2014b): Inicios del ajedrez en la prensa y en las publicaciones deportivas en España (1861-1915). Recorde: Revista de História do Esporte, $\mathrm{n}^{\circ} 7$, $11-34$.

BRASÓ, JORDI (2015): Thomas Arnold, Pere Vergés i els jocs organitzats. Els escacs, un projecte educatiu a l'Escola del Mar. Temps d'Educació, nº 49, 135-163.

BRUNET, JOSEP (1891): El ajedrez. Investigaciones sobre su origen. Barcelona: Lib. l'Avenç.

C. L. (1918): El concurso del real Club de Puerta de Hierro. Gran Vida, n ${ }^{\circ}$, 147148.

CALDERÓN, FERNANDO (2005): La mujer en la obra de Jean Jacques Rousseau. Revista de Filosofía, nº 30, 165-177.

Campeonato Mundial Femenino: Ajedrez. Revista Mensual (1930), nº 9, 172.

CAMPOAMOR, CLARA (1936): El derecho de la mujer. Madrid: Beltrán.

CARBAJOSA, CONCEPCIÓN; RIAÑO, CATALINA (2009): Proceso histórico de la incorporación de la mujer española a la práctica deportiva en el primer tercio del siglo XX. Citius, Altius, Fortius, nº 1, 23-47.

CARO, RODRIGO (1884): Días geniales o lúdricos. Sevilla: Imp. de El Mercantil Sevillano.

CASADEMUNT, M. (1908): Festes de la Garriga. Concurs de tir. Feminal, nº 18, 5.

CASTRO, VICENTE DE (1914): La educación física de la mujer. Gran Vida, nº 131, 117-118.

CASTRO, VICENTE DE (1915): Las artistas y el deporte. Gran Vida, nº 141, 47-50. 
CASTROVIDO, ROBERTO (1926): La casa del colegio internacional. Boletín de la Institución Libre de Enseñanza, n 800, 332.

Centro Auxiliar La Luz Andresense (1930): Centro Auxiliar La Luz Andresense. Barcelona, 8 nov. 1930.

Concurs de globos lliures (1908): Feminal, nº 14, 17.

CORTADA, ESTHER (2008): Feminisme i educació als inicis del segle XX. En VVAA ed. Pedagogia, política i transformació social (1900-1917): l'educació en el context de la fundació de l'Institut d'Estudis Catalans. Barcelona: Societat d'Història de l'Educació dels Països de Llengua Catalana, 199-226.

Curiosidades (25 diciembre 1894): Diario de Gerona, 14

DALBANNE, JOHANÉS (1913): Congreso Internacional de Educación Física. París 17, 18, 19, 20 de marzo de 1913. Gran Vida, n 118, 91-93.

DAMASIO, ANTONIO (2005): En busca de Spinoza. Neurobiología de la emoción y los sentimientos. Barcelona: Crítica.

DAMASIO, ANTONIO (2001): La sensación de lo que ocurre. Madrid: Debate.

DAMASIO, ANTONIO (2010): Y el cerebro creó al hombre. ¿Cómo pudo el cerebro generar emociones, sentimientos y el yo? Barcelona: Destino.

DE AGUSTÍN, MANUEL (1944): Torneo Internacional de Ajedrez. Margate 1939. Madrid: Jaque.

De información general española (19 agosto 1933), La Vanguardia, 2.

De toda España (1930): Ajedrez. Revista Mensual, nº 8, 160.

DELGADO, BUENAVENTURA (1981): La Escuela Moderna de Ferrer i Guàrdia. Barcelona: CEAC.

Deportes de nieve (1923): Gran Vida, nº 246, 367-370.

Deportes femeninos (1921): Gran Vida, nº 211, 19.

E. El Lawn Tennis y la dona (1907a): Feminal, nº 1, 18.

E. El sport y la dona-hipic (1907b): Feminal, nº 2, 18.

E. Els sports y la dona (1907c): Feminal, nº 4, 18.

E. El Gè concurs de Lawn-tennis (1908a): Feminal, nº 13, 18. 
E. L'últim concurs de lawn-tennis (1908b): Feminal, nº 20, 18.

El ajedrez femenino (5 noviembre 1932): Caras y Caretas, 112.

El concurso hípico de Madrid (1927): Gran Vida, nº 290, 263-266.

ELIAS, JOSEP (1907): Sport. Lawn-tennis. Feminal, nº 8, 18.

ELIAS, NORBERT; DUNNING, ERIC (1986): Deporte y ocio en el proceso de la civilización. México: Fondo de Cultura Económica.

ELIAS, NORBERT; DUNNING, ERIC (1992): Deporte y ocio en el proceso de la civilización. Madrid: Fondo de Cultura Económica.

ELSCHENBROICH, DONATA (1979): El juego de los niños. Estudios sobre la génesis de la infancia. Bilbao: Zero.

ENGELS, FREDERICK (1996): El origen de la família. Madrid: Fundamentos.

F. (1907): El feminisme a Catalunya. Feminal, no 9, 2.

F. (1908): La Federació de Pirineistes a Barcelona. Feminal, nº 12, 12.

FEIJOO, BENITO JERÓNIMO (1778): Defensa de las mujeres. Teatro crítico universal. Tomo I. Discurso XVI. Madrid: Real Compañía de Impresores y Libreros.

FERNÁNDEZ, ÁNGEL (1852): Los juegos de las diferentes edades, en todos los pueblos del mundo, desde la antigüedad más remota hasta nuestros días. Primera Serie. Juegos y diversiones de la infancia y de la juventud. Madrid: Biblioteca Universal.

FERNÁNDEZ, FRANCISCO (1864): Juegos y entretenimientos de las niñas. Madrid: Impr. del est. de Mellado.

FERNÁNDEZ, FRANCISCO (1862): Los Juegos de la Primera Edad. Primera Parte. Tomo III. Madrid: Est. Tip. de Mellado.

FERRER, FRANCISCO (1990): L'Escola Moderna. Explicació pòstuma i abast de l'ensenyament racionalista. Vic: Eumo.

FRANSA (1908): Feminal, nº 19, 15.

GARCÍA, JOSÉ (1934): El primer campeonato femenino de ajedrez... Estampa, $\mathrm{n}^{\circ}$ 351, 30. 
GARCÍA, PEDRO; FLORES, ZHANDRA; RODRÍGUEZ, ARMANDO; BRITO, PEDRO; PEÑA, RUBÉN (2008): Mujer y deporte. Hacia la equidad e igualdad. Revista Venezolana de Estudios de la Mujer, $\mathrm{n}^{\circ}$ 30, 63-76.

GARCÍA, PEDRO DE ALCÁNTARA (1879): Teoría y práctica de la educación y la enseñanza. Madrid: English y Gras, ed.

GARCÍA, PILAR (2008): Isabel de Borbón. Madrid: Alderabán Ediciones.

GARZÓN, JOSÉ ANTONIO (2010): Luces sobre el ingenio, el pionero libro del juego llamado marro de punta, de Juan Timoneda. Valencia: Centro Francisco Tomás y Valencia.

GARZÓN, JOSÉ ANTONIO (2010): Nuevos documentos relativos a la afición de los Reyes Católicos al ajedrez. En D'Ambrosio, L.; Rosino, A.; Schöne, S.; Stigter, J. ed., Festschrift zu Ehren Alessandro Sanvitos. Internationale Beiträge zur Geschichte und Bibliografie des Schachspiels, 251-271. Viena: Vindobona.

GOLEMAN, DANIEL (1996): Inteligencia emocional. Barcelona: Kairós.

GOLMAYO, MANUEL (3 mayo 1934): Ajedrez. Resultado del campeonato femenino de ajedrez. $A B C, 54$.

GÓMEZ-SANTOS, MARINO (1993): La Reina Victoria Eugenia. Madrid: Espasa Calpe.

GOUGES, O. (1989): The Rights of Woman. London: Pythia.

GUINART, RICARD (1933): Els campionats femenins d'escacs i la participació espanyola mundial. Els Escacs a Catalunya, no 72, 1409-1410.

GRAF, SONJA (1935): Una mujer ajedrecista. El Ajedrez Español, nº 12, 370-372.

HUGUET, MONTSERRAT (1989): La mujer española del siglo XVIII en la obra de Josefa Amar. B.I.L.E., $\mathrm{n}^{\mathrm{0}}$ 7, 43-57.

Información extranjera (1930): Ajedrez. Revista Mensual, nº 8, 151-152.

Intervención de las mujeres en el sport (1906): Gran Vida. Revista Ilustrada de sports, $n^{\circ} 43,1-5$.

IÑIGO, LUIS E. (2012): Breve historia de la Revolución Industrial. Madrid: Nowtilus. KARR, CARME (1907): La nostra finalitat. Feminal, nº 1, 2.

La aptitud de la mujer para el deporte (1927): Gran Vida, n² 288, 204-206. 
La Redacción. El Ajedrez y la Mujer (1935): El Ajedrez Español, nº 12, 369.

L'hivern de 1908 en les montanyes de Catalunya (1908): Feminal, nº 11, 15.

LAGARDERA, FRANCISCO [dir.] (1999): Diccionario Paidotribo de la Actividad Física y el Deporte. Barcelona: Paiotribo.

LAGARDERA, FRANCISCO Y LAVEGA, PERE (2003): Introducción a la praxiología motriz. Barcelona: Paidotribo.

LERENA, CARLOS (1976): Escuela, ideología y clases sociales en España. Madrid: Ariel.

LERENA, CARLOS (1983): Reprimir y Liberar. Madrid: Akal.

Los deportes apropiados para la mujer (1922): Gran Vida, nº 232, 309.

Los deportes en la juventud y su influencia en el Ejército (1915): Gran Vida, $\mathrm{n}^{0}$ 147, 251-252.

Los deportes en Santander (1918): Gran Vida, n 181, 209-214.

Los deportes y el feminismo (1925): Gran Vida, nº 266, 260-261.

MADAME D’AUTREMON (1932): Els Escacs a Catalunya, nº 61, 1157

MARCO, LUIS; OCHOA, EUGENIO (1895): Repertorio completo de todos los juegos. Madrid: Bailly-Bailliere.

MARTÍN, OCTAVIO (1989): Alcántara García y las escuelas de magisterio. Revista Interuniversitaria de Formación del Profesorado, n 4, 23-37.

MARTÍNEZ, V. (1865): Algunas consideraciones sobre ajedrez. El Museo Universal, $\mathrm{n}^{0} 25,199$.

MARX, KARL; ENGELS, FREDERICK (2012): El manifiesto comunista. Madrid: Nordica Libros.

MARX, KARL; ENGELS, FREDERICK (1981): La sagrada família. Madrid: Akal.

MARTÍNEZ, V. (1865): Algunas consideraciones sobre ajedrez. El Museo Universal, $n^{\circ} 25,199$.

Matx Velat-Puigcercós (1935): Els Escacs a Catalunya, nº 99, 1907-1908.

MCMILLEN, S. (2008): Seneca falls and the origins of the women's rights. New York: Oxford University Press. 
MENCHICK, VERA (1935): El Ajedrez Español, nº 13, 400-401.

MEYER, R. (1930): Un pueblo donde todo el mundo juega al ajedrez. Estampa, $\mathrm{n}^{\mathrm{o}}$ 137, 22-23.

MONTERO, ANTONIO (2009): La Ley de Instrucción Pública (Ley Moyano 1857). Cabás: Revista del Centro de Recursos, Interpretación y Estudios en materia educativa (CRIEME) de la Consejería de Educación del Gobierno de Cantabria, $\mathrm{n}^{\mathrm{o}}$ 1. Disponible en: http://revista.muesca.es/index.php/articulos/71-la-ley-deinstruccion-publica-ley-moyano-1857 [consulta el 2 de agosto de 2015].

MORAL, MARTA DEL (2007): Acción colectiva femenina republicana: las damas rojas de Madrid (1909-1911), una breve experiencia política. Hispania. Revista Española de Historia, n 226, 541-566.

MORALES, MARÍA LUZ (16 diciembre 1933), Ajedrez. Conferencia de María Luz Morales en el Club Barcelona, La Vanguardia, 12.

NADAL, JORDI (1975): El fracaso de la primera revolución industrial en España, 1814-1913. Barcelona: Ariel.

NAHARRO, VICENTE (1818): Descripción de los juegos de infancia, los mas propios a desenvolver sus facultades físicas, marales, y para servir de abecedario gimnástico. Madrid: Imp. que fue de Fuentenebro.

NASH, MARY (1983): Mujer, família y trabajo en España. Barcelona: Anthropos.

Notas locales (31 mayo 1925): La Vanguardia, 31.

Noticias (1896): Revista Internacional de Ajedrez, $\mathrm{n}^{0}$ 5, 70-72.

ORTEGA, JOSÉ (1943): Doña Isabel de Borbón. Infanta de España. Madrid: Aspas

PALUZÍE, JOSEP (1921): Manual de Ajedrez. Parte sexta (última). Miscelánea. Barcelona: s.e.

PAREJA, FÉLIX MARTÍN (1935): Libro del ajedrez, de sus problemas y sutilezas, de autor árabe desconocido. Madrid: Imp. de Estanislao Maestre.

Partida 883 (1932): Els Escacs a Catalunya, nº 60, 1127.

Partida 892. Campionat Femení de França (1932): Els Escacs a Catalunya, nº 61, 1155-1156.

Partida. Variante Primera (1930): Ajedrez. Revista Mensual, nº 6, 98-100. 
Partida núm. 17. Campeonato femenino de Francia, 1930 (1930): Ajedrez. Revista Mensual, $\mathrm{n}^{\circ}$ 2, 15.

PARLEBAS, PIERRE (2001): Juegos, deporte y sociedad. Léxico de praxiología motriz. Barcelona: Paidotribo.

PELAZ, DIANA (2011): Jaque a la reina: Cuando la mujer se convierte en un estorbo político. Miscelánea Medieval Murciana XXXV.

PLATÓN (1979): La República. Barcelona: Juventud.

PÉREZ, JOSÉ (1920). El ajedrez en la Argentina. Buenos Aires: Imp. Tixi y Schaffner.

PUIG, ESTEBAN (1933): Campionat Femení. Els Escacs a Catalunya, nº 67 , 12901291.

PUIG, NURIA; SOLER, SUSANA (2004): Mujer y deporte en España: estado de la cuestión y propuesta interpretativa. Apunts: Educació Física i Esports, nº 76 , 7178.

PUJADAS, XAVIER (2011): Del barrio al estadio. Deporte, mujeres y clases Populares en la segunda República, 1931-1936. En Pujadas, X. coord., Atletas y ciudadanos. Historia social del deporte en España, 1870-2010, 125-167. Madrid: Alianza Editorial.

PUJADAS, XAVIER (2010): Mujer y deporte: una larga carrera hacia la libertad (1880-2000), D MUJER, no 1, 15-20.

RAMOS, MARÍA DOLORES (2003). Historia de las mujeres, saber de las mujeres: la interpretación de las fuentes en el marco de la tradición feminista. Feminismo/s, $\mathrm{n}^{\circ}$ 1, 19-32.

RAMOS, MARÍA DOLORES (1999): Mujer, asociacionismo y sociabilidad en la coyuntura de 1898. Las afinidades con el fin de siglo europeo. En Sánchez, I.; Villena, R. coord., Sociabilidad fin de siglo. Espacios asociativos en torno a 1898, 73-100. Cuenca: Publ. de la Universidad de Castilla-La Mancha.

REMENTERÍA, MARIANO DE (1839): Manual Completo de Juegos de Sociedad o tertúlia, y de prendas. Madrid: Imp. de Don Norberto Llorenci.

Resultados del torneo de Margate (6 mayo 1936): ABC, 54.

REY, RAMÓN (9 septiembre 1932): El ajedrez y la mujer. La Vanguardia, 10. 
REY, RAMÓN (16 diciembre 1932): Sección de Ajedrez. Ajedrez femenino: La Vanguardia, 13.

REY, RAMÓN (26 mayo 1933): Sección de Ajedrez. De Actualidad, La Vanguardia, 11.

REY, RAMÓN (2 febrero 1934a): Sección de Ajedrez. Actividad sin tregua, La Vanguardia, 12.

REY, RAMÓN (4 mayo, 1934b): Sección de Ajedrez. El Ajedrez en Madrid, La Vanguardia, 12.

ROBIN, PAUL (1981): Manifiesto a los partidarios de la educación integral. Barcelona: Pequeña Biblioteca Calamvs Scriptrorivs.

SAGARRA (20 diciembre 1932): Notas Gráficas. Campeonato Femenino de Ajedrez, La Vanguardia, 1-2.

SÁNCHEZ, ADOLFO (1982): Filosofía y economía en el joven Marx: Manuscritos de 1844. México: Grijalbo.

SÁNCHEZ, RAÚL; RIVERO, ANTONIO (2013): Govern mentality in the origins of European female PE and sport: the Spanish case study (1883-1936). Sport, Education and Society $\mathrm{n}^{\circ}$ 4, 494-510.

SÁNCHEZ-OCAÑA, JAVIER (1935): Barcelona, paraíso de los jugadores de ajedrez. Cuarenta clubs; veinte mil aficionados. Estampa, nº 371, 11-14.

SANFELIU, LUZ (2008): Del laicismo al sufragismo. Marcos conceptuales y estrategias de actuación del feminismo republicano entre los siglos XIX y XX. Revista de Historia Contemporánea, no 7, 59-78.

SANTASUSAGNA, ANTONIO (1932): Campionat Infantil Individual (1932). Els Escacs a Catalunya, ${ }^{\circ}$ 59, 1094-1098.

SANTORO, ÓSCAR (2010): ¿Es el ajedrez un deporte? ISDe Sports Magazine-Revista de entrenamiento, $\mathrm{n}^{\circ}$ 2(7), 1-6.

SANTOS, PADRE (1876): Juegos de los niños en las escuelas y colegios. Madrid: Saturnino Calleja.

SANZ, JOSÉ (3 febrero 1933a): Ajedrez. Asamblea en Barcelona. Luz, 14

SANZ, JOSÉ (14 abril 1933b): Ajedrez. Comentarios al reciente campeonato. Luz, 14.

SANZ, JOSÉ (9 febrero 1934): La mujer y el ajedrez. Luz, 14. 
Sección de partidas (1936): Ajedrez Español, nº 18, 50-53.

SERRANO, LEONOR (1992): El treball intel-lectual i el treball manual de la dona moderna. En González-Agàpito, J. coord., L'Escola Nova Catalana. 1900-1939. Vic: Eumo, 123-125.

SIMÓN, JUAN ANTONIO (2009): El papel de la mujer en el origen y desarrollo del deporte en España, 1900-1939. Actas del primer Congreso Internacional. Las mujeres en la esfera pública. Filosofía e historia contemporánea. Madrid: Compañía Española de Reprografía y Servicios, S.A., 22-102.

Storbeck, la ciudad del ajedrez (1851): La Ilustración: periódico universal, nº 6, 48.

STUART, J. (2008): La esclavitud femenina. Madrid: Artemisa.

TARTAKOWER, SAIVELLY (1929): Errores instructivos. Ajedrez. Revista Mensual, $n^{0} 1,3-6$.

TARTAKOWER, SAIVELLY (1930). Impresiones de Niza. Ajedrez. Revista Mensual, $n^{0} 5,73-75$.

TERMCAT, CENRE DE TERMINOLOGIA (2010). Diccionari general de l'esport. Barcelona: TERMCAT, Centre de terminología.

TORNÉ, AMPARO (2002): Luces y sombras en el camino hacia una escuela coeducativa. En VVAA ed., Mujer y educación. Educar para la igualdad, educar desde la diferencia, 169-183. Barcelona: Graó.

TORREBADELLA, XAVIER (2013): Anotaciones al balonmano en el contexto histórico del deporte en España (1900-1939). E-balonmano.com: Revista de Ciencias del Deporte, $\mathrm{n}^{\circ}$ 2, 115-134.

TORREBADELLA, XAVIER (2016): Fútbol en femenino. Notas para la construcción de una historia social del deporte femenino en España, 1900-1936. Investigaciones Feministas, no $7(1)$, 313-334.

TORREBADELLA, XAVIER (2011): La educación física y la actividad gimnásticodeportiva de las mujeres a partir de la bibliografía especializada del siglo XIX. Arenal, $\mathrm{n}^{0}$ 1, 147-179.

TORREBADELLA, XAVIER; BRASÓ, JORDI (2014): El joc del 'rescat' a Catalunya. Un projecte educatiu a l'Escola del Mar de Pere Vergés. Temps d'Educació, nº 47, 191-212.

TRISTAN, FLORA MME (1844): Union Ouvrière. Paris et Lyon: Chez tous les libraires. 
VARELA, JULIA (2011): Mujeres con voz propia: Carmen Baroja y Nessi, Zenobia Camprubí Aymar y María Teresa León Goyori. Madrid: Morata.

VERGÉS, PERE (1933): Els escacs a l'Escola del Mar. Els escacs a Catalunya, nº 71, 1388-1389.

VICENTE-PEDRAZ, MIGUEL; BROZAS-POLO, MARÍA PAZ (2014): El 'discurso sobre la Educación Física y moral de las mujeres' (1790) de Josefa Amar y Borbón: feminidad y el arte de gobernar el cuerpo en la ilustración española. Movimento, $\mathrm{n}^{\circ}$ 2, 799-818.

VIDAL (24 de julio 1926): Mademoiselle Marie Jeanne Frigard, La Voz, 8.

VILAR, JUAN-BAUTISTA (1990): La primera revolución industrial española: 18271869. Madrid: Istmo.

VINYOLES, TERESA; MARTÍN, SUSANNA; CHALAUX, LÍDIA (2003): La rueca y la espada. Las mujeres medievales, la guerra y la paz. En Nash, M. y Tavera, S. coords., Las mujeres y las guerras, 73-82. Barcelona: Icaria.

WADE, MARGARET (2003): La mujer en la Edad Media. San Sebastián: El País.

WESTERVELD, GOVERT; GARZÓN, JOSÉ ANTONIO (2004): La reina Isabel la Católica, su reflejo en la dama poderosa de Valencia, cuna del ajedrez moderno y origen del juego de damas. Valencia: Conselleria de Educación, Cultura y Deporte.

WOLLSTONECRAFT, MARY (1792): A Vindication of the Rights of Woman. London: J. Johnson. 